\title{
Aortic Valve replacement via Right Anterior mini-Thoracotomy: a simpler minimal
}

\author{
Gabriele Tamagnini ${ }^{1}$, Raoul Biondi ${ }^{1}$, Gabriella Ricciardi ${ }^{2}$, Jose Zulueta ${ }^{1}$, Massimiliano \\ Foschi $^{3}$, and Mauro Del Giglio ${ }^{1}$ \\ ${ }^{1}$ Villa Torri Hospital \\ ${ }^{2}$ Leiden Universitair Medisch Centrum \\ ${ }^{3}$ SS Annunziata Hospital
}

November 6, 2020

\begin{abstract}
Minimally Invasive Aortic Valve Replacement is not just a matter of the incision, but it should be rather considered as a holistic approach to minimize surgical trauma: our technique aims to reproduce the gold-standard conventional procedure in terms of safety, effectiveness and operative time through a small and different incision; furthermore with a thorough optimization of the procedure, the surgical time can be at least equal to the classic approach. We introduced our approach about 10 years ago; after more than 1000 aortic valve replacement via right anterior mini-thoracotomy, we developed with some tips and tricks in the attempt of turning this technique into our choice approach. Indeed, it is our belief that a highly standardized technique is pivotal to perform a safe and valuable procedure.
\end{abstract}

\section{Title}

Aortic Valve replacement via Right Anterior mini-Thoracotomy: a simpler minimal

\section{Running head}

Making the standard approach smaller

Authors: Gabriele Tamagnini ${ }^{1}$, MD, Raoul Biondi ${ }^{1}, \mathrm{MD}$, Gabriella Ricciardi, MD ${ }^{2}$, Josè Luis Zulueta ${ }^{1}$, MD, Massimiliano Foschi ${ }^{3}$, MD and Mauro Del Giglio ${ }^{1}, \mathrm{MD}, \mathrm{PhD}$

\section{Institutions and affiliations:}

1 Cardiovascular Department, Villa Torri Hospital - Via Quirico Filopanti, 12 - Bologna - Italy

2 Department of Cardiac Surgery, Leiden Universitair Medisch Centrum, Leiden, The Netherlands

3 Cardiac Surgery Department, "SS. Annunziata" Hospital - Via Dei Vestini - Loc. Colle Dell'Ara, Chieti Italy

Classification: aortic valve replacement, minimally invasive surgery

Article Word count: 2027

\section{Corresponding Author}

Gabriele Tamagnini, M.D. - Villa Torri Hospital - via Quirico Filopanti, 12 - Bologna - Italy

Email: gabriele.tamagnini@gmail.com 


\title{
Funding: none
}

\section{Conflict of Interest}

The authors whose names are listed above certify that they have NO affiliations with or involvement in any organization or entity with any financial interest (such as honoraria; educational grants; participation in speakers' bureaus; membership, employment, consultancies, stock ownership, or other equity interest; and expert testimony or patent-licensing arrangements), or non-financial interest (such as personal or professional relationships, affiliations, knowledge or beliefs) in the subject matter or materials discussed in this manuscript.

\section{Data availability statement}

Data sharing is not applicable to this article as no new data were created or analysed in this study.

\section{Informed Consent}

The production of this paper and the collection of those retrospective data were approved by the local ethics committee. As a surgical technique paper, the Authors requested the consent requirement to be waived because the research involves no more than minimal risk to the subject and the waiver will not adversely affect the rights and welfare of the subjects.

\section{International Review Board Statement}

As a surgical technique paper, the Authors requested the IRB approval to be waived because the manuscript is not a trial and it involves no more than minimal risk to the subject and the waiver will not adversely affect the rights and welfare of the subjects.

\begin{abstract}
Minimally Invasive Aortic Valve Replacement is not just a matter of the incision, but it should be rather considered as a holistic approach to minimize surgical trauma: our technique aims to reproduce the goldstandard conventional procedure in terms of safety, effectiveness and operative time through a small and different incision; furthermore with a thorough optimization of the procedure, the surgical time can be at least equal to the classic approach. We introduced our approach about 10 years ago; after more than 1000 aortic valve replacement via right anterior mini-thoracotomy, we developed with some tips and tricks in the attempt of turning this technique into our choice approach. Indeed, it is our belief that a highly standardized technique is pivotal to perform a safe and valuable procedure.
\end{abstract}

\section{Central Message}

Minimally Invasive AVR (Mi-AVR) should minimize surgical trauma to reach the gold-standard conventional procedure in terms of safety, effectiveness and operative time, although the different approach adopted.

\section{Article}

Minimally Invasive aortic valve replacement gained growing consent in the last years [1] based om associated minimal surgical trauma, faster rehabilitation, better pain control and patient compliance. Since the beginning of our experience, we focused on simplification and optimization of the technique to make the MiAVR as versatile and as reproducible as the conventional median sternotomy approach. Our aim was to reach the result of the gold-standard approach through a smaller incision[2], [3]. The key elements to realize a true minimally invasive procedure include sparing the right mammary artery, avoiding rib fractures, adopting a total central cannulation[4] - of paramount importance to improve both perfusion and drainage while on cardiopulmonary bypass (CPB) - and a short operative time to make that procedure minimally invasive from a biological point of view too. We strongly believe that minimally invasive surgery should spread across the developing country as well, because it holds advantages in terms of blood transfusion rate, intubation and Intensive Care Unit (ICU) times and pain control: a simple technique would be extremely reproducible even in Centres working on a limited budget. 
After more than 1200 RAT-Aortic Valve Replacement, we were able to optimize our technique (Central Picture). The patient lies in supine decubitus and an inflatable bag is placed behind the right scapula to tilt the thorax toward the left side. Double lumen intubation is provided to exclude the right lung. The second or third intercostal space is opened through a 5-cm skin incision, paying attention to preserve the right mammary artery and avoiding any rib damage. We do not perform routinely a CT-scan to plan the operation: the chest X-ray and the aortography during the coronary artery catheterization, in our experience, are reliable to highlight the presence of worrisome aortic calcification or unusual anatomic variants; on the other hand, we do recognize the value of a pre-operative CT-scan when starting a MiAVR program: in the first cases, that allows to rule out patients who are more demanding in terms of surgical skills [5]. The correct site to carry out the thoracotomy is identified looking for position of the angle of Louis and, from there, that of the third rib: we perform a $5 \mathrm{~cm}$ long incision along its edge, starting at a distance of $5 \mathrm{~cm}$ from the jugular-xiphoid line (Fig. 1). The third intercostal space (just below the incision line) is most of the times the appropriate space to gain access to the thorax: the right superior pulmonary vein should be appreciated in the middle of the surgical field. However, if the exposition seems to be not adequate for the patient's individual anatomy, it is nonetheless easy to switch from the 3rd to the 2 nd space upon the same incision. To further improve the view throught the intercostal space, a soft tissue retractor and an appropriate spreader are adopted. The pericardium is opened as medial as possible, almost as in median sternotomy, keeping a safe distance from the phrenic nerve. This incision in extended up to the pericardial reflection and down towards the diaphragm, to pursue the best right atrial exposure. This correct exposure is critical to secure an easy procedure. Then, to further improve the exposure and to cope with the anatomical variances as well, we use three pericardial retractions, making a deep figure-of-eight stitch with a strong and thick wire: one is positioned at the level of the right superior pulmonary vein, the second as cranial as possible and the last as caudal as possible from the first respectively. Those stitches are passed through the thoracic wall with an EndoClose and pulled to bring the aortic root closer to the thoracotomy, especially in case of deep and left-sided aorta (Fig. 2). Our technique strictly relies on a totally central and direct arterial and venous cannulation. Central cannulation not only reduces surgical trauma, but also avoids the potential complications related to the femoral approach, at the same time providing a more physiological perfusion flow and better venous drainage. Furthermore, retrograde arterial flow demonstrated a strong correlation with femoral and aortic dissection, ipsilateral limb ischemia and cerebrovascular and renal events. For arterial cannulation, the EOPA cannula is inserted through a double purse-string suture placed at the pericardial aortic (Fig. 3), while a three-stage 29-Fr cannula is placed in the right atrium for the venous drainage. Both the optimal view and control of the target spot are critical to perform a harmless aortic cannulation: one useful trick is to push the aorta down and towards the operating surgeon using sponge and forceps. Regarding the venous cannulation, as it might be difficult to engage the inferior cava vein with a straight cannula, we use a metal spindle (e.g. the one included in the intracavitary venting small cannula) to obtain a rounded shaped venous cannula (Fig. 4). Aside the hydrodynamic advantage, the venous cannulation through the right appendage may further improve the exposure. Indeed, we use a snare that is strongly and safely fastened around the venous cannula next to the right appendage, which is also passed through the thoracic wall: a gentle pull on this snare will move the right atrium away, improving the exposure of the aortic root. The left ventricle is vented via a small cannula inserted through the right upper pulmonary vein into the left ventricle. Continuous carbon dioxide flow at a rate of $3 \mathrm{~L} / \mathrm{min}$ is routinely delivered throughout the procedure. A Chitwood-DeBakey aortic cross clamp is placed at right below the origin of the innominate, avoiding the dissection between the ascending aorta and the pulmonary trunk. A separate small incision just below the lateral clavicular side, measuring less than $1 \mathrm{~cm}$, is used to insert the clamp across the transverse sinus, so as to achieve the least crowded operative field (Fig. 5). Blood cold cardioplegia is administered in an antegrade fashion. Blood cold St.Thomas Cardioplegia, which is enriched with procaine, is administered in an antegrade fashion to guarantee an ischemic time of about $40 \mathrm{~min}$. The use of sutureless bioprostheses or a running suture technique for the implant usually bewares the need for a second cardioplegic infusion. In case of significant aortic regurgitation, part of the cardioplegic solution is delivered antegradely to arrest the heart, while the rest of the dose is admnistered directly into the coronary ostia: the left main stem ostium can be easily reached through the aortotomy, albeit the identification of the access to the right ostium 
might require the use of a small dentist looking glass; whether a second shot is needed, the coronary ostia are used for this purpose. We routinely use a transverse aortic incision to excise the diseased aortic valve en bloc (Fig.6). Thus, either a sutureless or a sutured prosthesis can be implanted. In case of a sutured prosthesis, the preferred technique forecasts three running 2/0 Prolene $(120 \mathrm{~cm})$ sutures with $3 / 4$ big steps along each sinus: according to our experience, this technical tool warrants better hemodynamics, faster implant and marginal risk of paravalvular leaks. In addition, we use a silked thread placed at the middle of the suture to apply counter traction and stretch the suture properly, thus reducing the occurrence of paravalvular leaks to a greater degree. The dearing is done gently filling the heart, with the surgical table in anti-Trendelenburg position, and in the meanwhile the aortotomy is closed using a single or double 4/0 Prolene suture. Ventricular pacing wires are placed on the right ventricle. The aortic clamp is removed and the patient is weaned from cardiopulmonary bypass; the intracavitary venting line is removed as soon as the echocardiographic monitoring demonstrated the absence of bubbles within the cardiac chambers. Finally, it is imperative to realize an exhaustive hemostasis in the relatively narrow space of the surgical access. Therefore, we use PTFE pledgets on every purse-string.

\section{References}

1] J. Lamelas and T. C. Nguyen, "Minimally Invasive Valve Surgery: When Less Is More.," Semin Thorac Cardiovasc Surg, vol. 27, no. 1, pp. 49-56, 2015.

[2] E. Mikus, S. Calvi, M. Panzavolta, J. Luis Zulueta, L. Dozza, A. Cavallucci, and M. Del Giglio, "Right Anterior Mini-Thoracotomy: A 'New Gold Standard' for Aortic Valve Replacement?," J. Heart Valve Dis. , vol. 24, no. 6, pp. 693-698, Nov. 2015.

[3] E. Mikus, S. Turci, S. Calvi, M. Ricci, L. Dozza, and M. Del Giglio, "Aortic valve replacement through right minithoracotomy: is it really biologically minimally invasive?," The Annals of Thoracic Surgery, vol. 99, no. 3, pp. 826-830, Mar. 2015.

[4] K. Bedeir, M. Reardon, M. Ramchandani, K. Singh, and B. Ramlawi, "Elevated Stroke Risk Associated With Femoral Artery Cannulation During Mitral Valve Surgery.," Semin Thorac Cardiovasc Surg, vol. 27, no. 2, pp. 97-103, 2015.

[5] M. Del Giglio, G. Gabriele, R. Biondi, and M. Di Mauro, "A simpler minimal," Journal of Cardiac Surgery , vol. 106, no. 6, pp. 1782-1, Sep. 2020.

Central Picture: Our usual approach: Right Anterior mini-Thoracotomy and total central cannulation.

Fig. 1: Site selection for the thoracotomy

Fig. 2: The configuration of the three pericardial stitches to optimize the exposure

Fig. 3: The direct arterial cannulation

Fig. 4: The rounded shaped venous cannula to engage easier the inferior cava vein

Fig. 5: The cross-clamp manoeuvre: a separate skin incision gives a less crowded surgical field

Fig. 6: The native aortic valve from the surgeon's point of view 


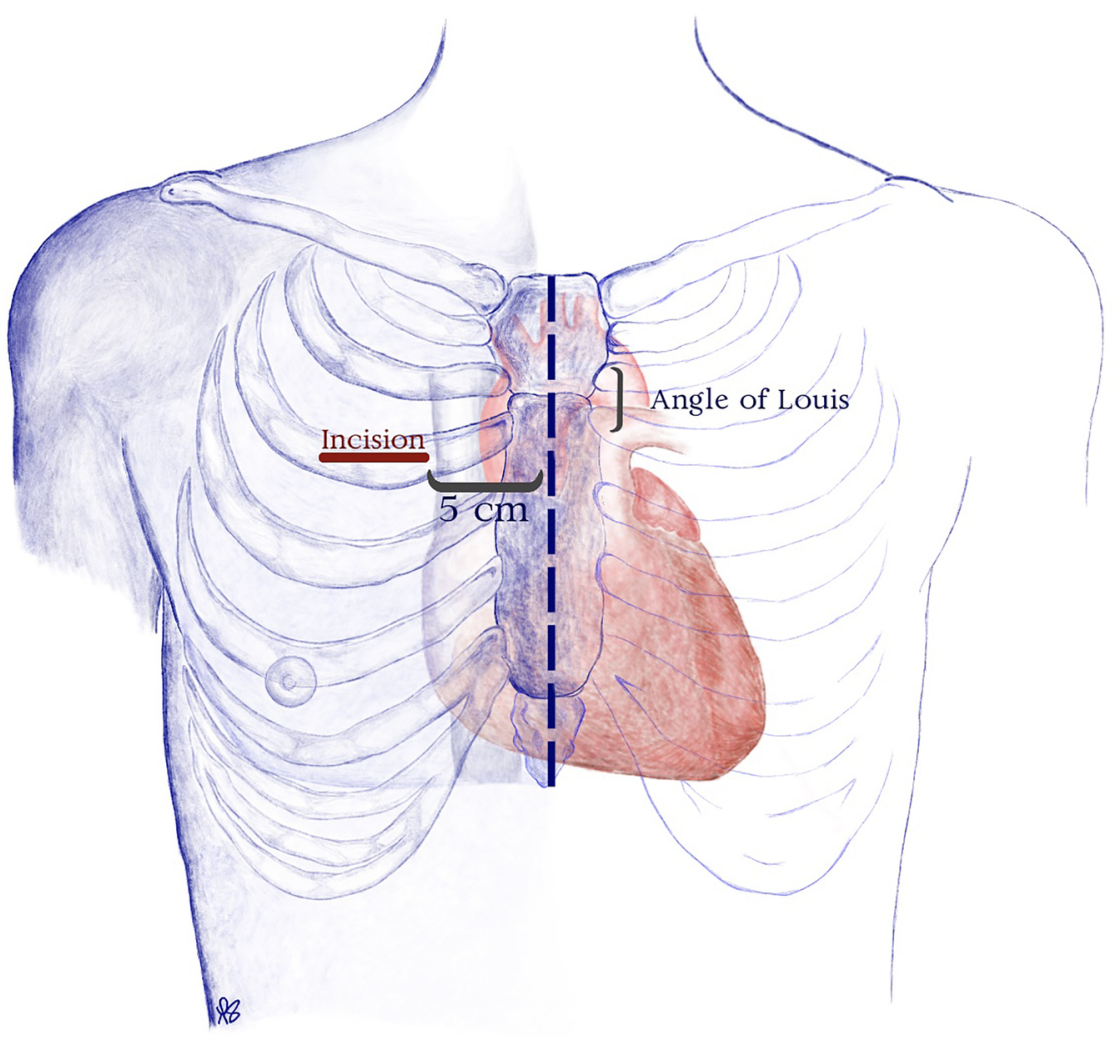



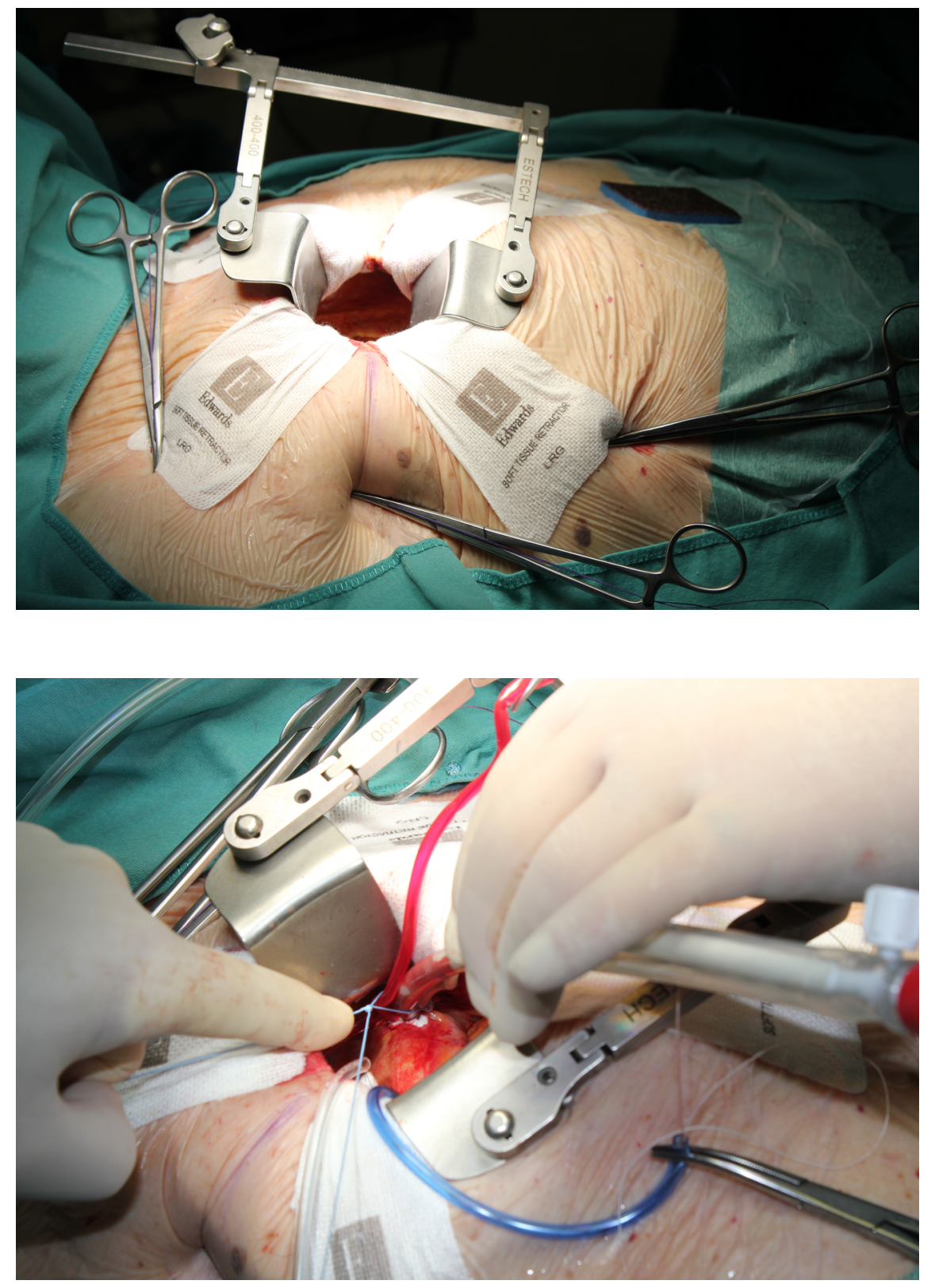

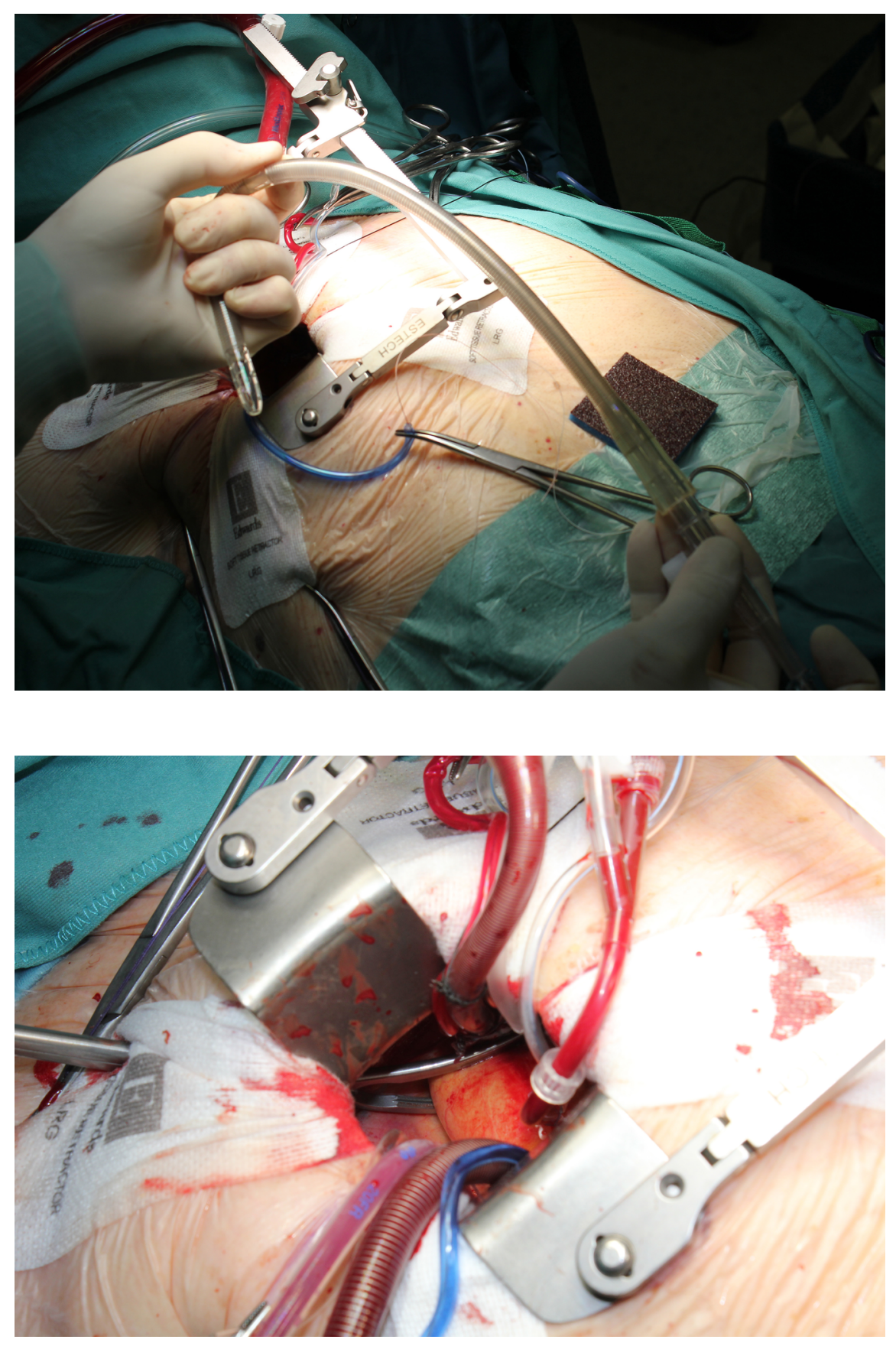


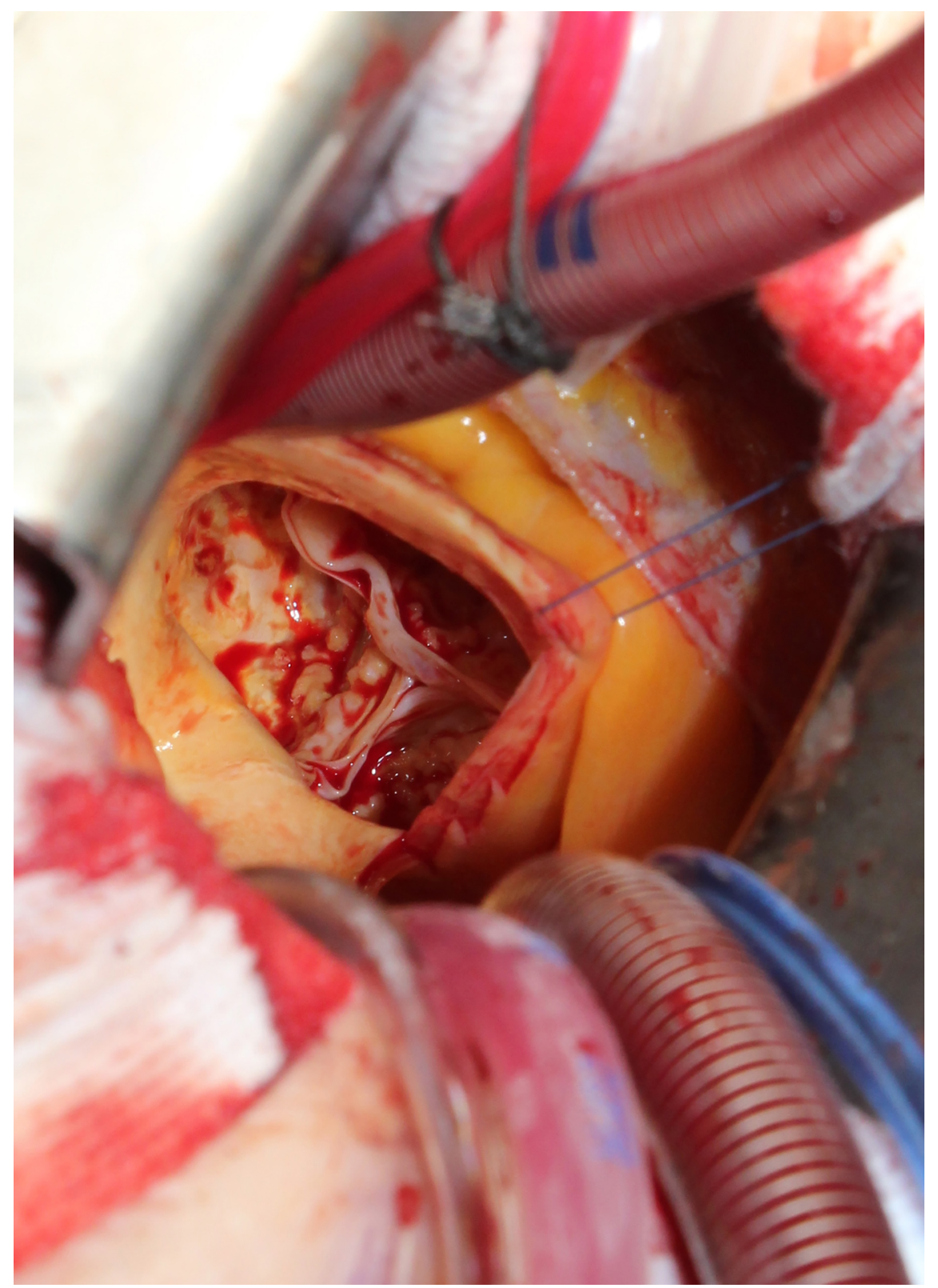

